\title{
Reasentamiento colectivo postdesastre como alternativa de habitar
}

Post-disaster collective resettlement as an alternative to inhabiting

Jeison Andrés Hincapié Rodríguez

Jeian91@gmail.com

Fundación Universitaria San Alfonso

Enviado 16/04/2021 - Aceptado 24/06/2021

Hincapié Rodríguez, J.A. (2021). "Reasentamiento colectivo postdesastre como alternativa de habitar". En Proyección: estudios geográficos y de ordenamiento territorial. Vol. XV, (29). ISSN 1852 -0006, (pp. 8 - 29). Instituto CIFOT, Universidad Nacional de Cuyo. Mendoza. 


\begin{abstract}
Resumen
Los eventos asociados a fenómenos socio-naturales generan situaciones que modifican la vida de los habitantes de un asentamiento de viviendas por autoproducción en cuanto a su cotidianidad, construcciones espaciales y sociales quienes, por medio de los modos de habitar, adecuan el espacio físico y social para desarrollar el habitar. En tal forma, a partir de dichas externalidades, se implementan nuevas formas de ocupar, ya que el hábitat implica relaciones sociales y con el medio físico para su producción.

Esto conlleva a que se realicen intervenciones por parte de la administración, tales como traslados poblacionales, los cuales se han centrado en dar respuestas parciales, que no contribuyen a disminuir la vulnerabilidad, pues destacan a la vivienda como un producto final. En tal forma, la investigación propone lineamientos para el reasentamiento colectivo, entendido como la autoproducción del espacio habitable de una colectividad, en la que se tejen relaciones de vecindad, comunitarias e imaginarias, conservando los códigos y modos de habitar, los cuales se construyen de manera transversal a partir de la construcción del hábitat, dando relevancia a las formas de intervención por parte de los habitantes.
\end{abstract}

Palabras clave: Autoproducción, Hábitat, Planeación urbana, Reasentamiento, Riesgo.

\title{
Abstract
}

The events associated to socio-natural phenomena generate situations that modify inhabitants' life at self-production housing settlement, in relation to their daily life, spatial and social constructions. They, by different ways of inhabiting, adapt both physical and social space to develop their own way of inhabiting. Thus, from these externalities, new forms of occupy are implemented, since the habitat implies relations with the physical and social environment, for its production.

This leads to state measures, such as population transfers, which are only focused on providing partial solutions that do not contribute to reduce vulnerability, since they highlight housing as a final product. In this way, the research proposes guidelines for collective resettlement, understood as the self-production of the habitable space of a community, in which neighborhood, community and imaginary relationships are woven, conserving the codes and ways of living, which are built in a transversal way from the construction of the habitat, giving relevance to the forms of intervention by the inhabitants.

Keywords: Collective resettlement, Habitat, Urban planning, Self-production, Risk. 


\section{Introducción}

Los fenómenos de origen socio-natural tales como, deslizamientos e inundaciones, transforman las realidades habitacionales producidas desde la vivienda informal, por lo que se procura la atención del Estado de diferentes formas, una de ellas a partir del reasentamiento poblacional, el cual evita el riesgo alto, por medio del traslado físico. Sin embargo, el reasentamiento no se desarrolla de manera integral, pues a los actores sociales se les toma como pasivos, lo que reduce su participación, mientras que prima la inmediatez en la gestión de soluciones habitacionales, con un sesgo hacia la estructura netamente física de las unidades de vivienda.

Así mismo, se omiten las dimensiones sociales, económicas y espaciales, a partir de los modos de habitar, que se han gestado desde asentamientos por autoproducción, lo cual tiene repercusiones en la fase de postreasentamiento, implicando nuevas vulnerabilidades para la sustentabilidad de los barrios, así como sociales, teniendo en cuenta el no habitar, como un nuevo fenómeno urbano de desterritorialización.

Por ello, en el presente escrito se indaga sobre la importancia del hábitat como un componente integral, en el que tiene cabida la vivienda y el entorno, como elementos fundamentales del programa de reasentamiento colectivo, lo que promoverá la apropiación socioespacial, identidad, resignificación del espacio vivido y habitado, así como la producción de vivienda que se debe acoplar a las necesidad y entornos, fortaleciendo la participación decisiva, economía solidaria y colectividad.

Como objetivo central del documento, se proponen lineamientos para la intervención del reasentamiento, los cuales se obtuvieron a partir del trabajo con grupos focales, realizado con habitantes que han sido reasentados, por lo que se gestan desde una mirada comparada en los efectos funcionales, habitacionales y de la participación comunitaria, en dimensiones económica, social, política y organizacional, para promover la sostenibilidad en el tiempo.

Se destacan dos dimensiones de análisis, la primera es la físico-espacial, a partir de los niveles de apropiación, riesgo, formas de habitar en el barrio que se reasenta y luego ver las trasformaciones en materia sociocultural, desde la ocupación de las nuevas viviendas, es decir del espacio vivido, desde una metodología cualitativa, dado que reúne la comprensión del contexto, interpretación de lo expresan los habitantes participantes y la posibilidad de un diseño de lineamientos apoyado en la observación y el trabajo de campo en los barrios. 
Este diseño de lineamientos se ejecutó entre enero y marzo de 2020 en la ciudad de Bogotá, Colombia, para lo cual se realizaron visitas exploratorias, así como de aplicación de instrumentos y co-creación de la propuesta final, a partir de la interpretación de las necesidades y relacionar del habitar. Ello se logra por medio de la aplicación de métodos de investigación social, tales como observación participante y grupos focales, fundamentales para la resignificación de programa de reasentamiento, mediante preguntas con las cuales se infirió las condiciones y atributos de los asentamientos previos al reasentamiento, así como las necesidades sentidas en los barrios en los que viven actualmente, en torno al hábitat integral, con el ánimo de revisar cómo se pueden fortalecer, evitando el retorno a los sitios con riesgo y reducir nuevas vulnerabilidades en los barrios reasentados.

El documento se desarrolla en tres partes; el primero describe el marco referencial para el reasentamiento colectivo, bajo las dimensiones del hábitat, comprendiendo las tensiones que se desatan por la intervención. El segundo aparte, describe los resultados del trabajo de campo en el barrio Colores de Bolonia I, Bogotá-Colombia, su análisis se desarrolla sobre las características físicas de las unidades habitacionales, su ubicación en la ciudad y la manera en cómo fue gestionado, lo que luego es tenido en cuenta para la formulación de lineamientos, así como el trabajo con parte de sus residentes.

En el tercer apartado se comprende la producción simbólica de las viviendas, redes sociales y vínculos reglados por imaginarios y la memoria, incluyéndolos en los lineamientos propuestos en este documento que, consideran la triada: individuo, sociedad, naturaleza, a través de la significación del espacio, para múltiples encuentros y el sentido del apego; finalmente, se describen algunas conclusiones y recomendaciones para entes territoriales que, deben desarrollar procesos de traslado poblacional, por motivos de riesgos socionaturales.

\section{Reconfiguración del habitar por reasentamiento}

Este primer apartado destaca la importancia de las relaciones entre el ser humano y el medio donde habita, lo que implica gestión comunitaria del riesgo frente a nuevos escenarios, ya sea por fenómenos naturales o por la ruptura en las relaciones diversas, pues habitar implica dar respuesta material al sentido de la vida urbana, para este caso. De tal manera, cuando se realizan reasentamientos poblacionales, hay dificultades en la propuesta y materialización de un proyecto urbano común, teniendo en cuenta la separación de la población, así como la limitación de prácticas cotidianas colectivas e individuales. 
Estas condiciones que marcan la movilización forzada en el espacio habitado implica entender que, el habitar es un fenómeno cultural, aunque desde los programas institucionales se ve a la vivienda, como el centro de la atención y no un componente en las diversas redes del asentamiento. Desconociendo también que, las unidades habitacionales que se produjeron estuvieron concebidas a partir de la modelación que aporta el medio, así como de las cosmovisiones individuales y colectivas que, permitieron la mejora de las condiciones del hábitat, entendido como espacio vivido en el que se gestan prácticas sociales y acciones de la creación del espacio y la vida social.

Por lo que la pérdida del territorio marca profundamente, la existencia de los individuos en el mundo, así como de las relaciones que, pueden ser sociales, económicas, físicas, políticas, entre otras. A otra escala, la ruptura del hogar mismo, como un esquema de cuidado y protección a los miembros de la familia, también implica a objetos y tipologías de la vivienda en su interior, las que han sido concebidas, en la medida de las posibilidades, para desarrollar unos modos de vida.

Por esta razón, menciona Arrieta (2005), que las zonas declaradas como de alto riesgo, al ser ocupadas quedan cargadas simbólicamente, no con el hecho de ocupar, sino con los modos de habitar. Entonces, las múltiples acciones de los sujetos en el habitar, tejen estas redes sociales que marcan los espacios en los que las personas han construido sus historias y formas de ver el mundo.

Por su parte Partridge (2000), ve que el reasentamiento no es solo físico, en cuanto a la vivienda, infraestructura o adecuación del espacio ya que, implica la recuperación del sentido comunitario, el valor humano, las formas de producción comunitaria, por lo que una intervención promoverá un cambio en las relaciones que se tejieron frente a un espacio habitacional.

Mientras que autoras como, Echeverría y Rincón (2000), ven que en el territorio se identifican proyectos, asociados a los metros cuadrados requeridos, sin que se tengan en cuenta la condición de los habitantes, por lo que no se identifica el territorio desde los códigos de quienes lo interpretan, es decir de quienes no solo lo ocupan, sino que desarrollaron sentimientos y su modo de vida en él. Puesto que, el desarraigo puede entenderse como el desprendimiento de la participación en la producción de su hábitat, compuesto por los lazos de comunidad y la historia con que se construyó.

Luego, el proceso de reasentamiento implica no habitar el lugar, lo cual no se tiene en cuenta para tratar las compensaciones, pues la existencia de un hábitat surge exclusivamente desde quienes lo sienten y le otorgan ese carácter. Ante ello, Correa (1997), entiende una modificación en las reacciones psicológicas ante el 
reasentamiento, manifestado en actitudes de rechazo, oposición e indiferencia, así como en sentimientos de pérdida de la vivienda que, no se podrá compensar en su integralidad.

Lo que Cernea (1995), considera como una manera de desorganización social que ponen en otra clase de riesgo a la población, de empobrecimiento social, cultural y económico, que desmiembran las pequeñas economías; por ejemplo, prohibiendo las ventas al interior de los nuevos conjuntos habitacionales, que además tienen implícito un rechazo a la otredad. Como afirma Serje (2011), no se pueden desconocer las posibilidades de beneficios que, para muchas familias puede traer un proceso de reasentamiento.

Para Hurtado (2010), el reasentamiento es una experiencia que involucra la transformación de la cotidianidad, debido al traslado, que tiene por fin mejorar la calidad de vida y construir un hábitat digno. Lo que implica transformaciones en las dimensiones de la vida: social, territorial, ambiental, física, jurídica, económica, organizativa, discursiva o cultural, psicológica, político-administrativa, ampliando la perspectiva que se dispone desde la ley 388 de 1997, en la que no se hace mayor referencia para el proceso, más que la concepción física y administrativa, en la que no se dan mayores lineamientos para los municipios, con lo cual estos entes administrativos no poseen herramientas considerables para abordar el reasentamiento postdesastre.

Luego, para Hurtado y Chardón (2012), el ejercicio del reasentamiento debe involucrar la transformación de la cotidianidad, a partir de un traslado poblacional definitivo bajo situaciones complejas tantos individuales como colectivas, luego de haber estado en un hábitat transitorio, a partir de un arrendamiento; además, se da fuera de un círculo de permanencia que pretende la calidad de vida y consolidación de un hábitat digno.

Por ello, los modos de habitar se convierten en un elemento clave en la disposición de los roles, de las redes económicas y sociales de acuerdo con la diferenciación en la ubicación, y formas de participación comunitaria en la producción del asentamiento. Es así como el hábitat es la suma del entorno geográfico, observando las dimensiones sociales, económicas y políticas de la vida ya sea de manera individual o desde la colectividad que, se modifica con un traslado de su vivienda, lo que implica las dimensiones subjetivas del espacio, Ortiz (2004), los considera como la vivencia y percepción del individuo.

Retomando a Echeverría (2009), la construcción del espacio es derivado de los habitantes y de las acciones de los agentes que influyen en su crecimiento y ocupación que, en algunos casos se realiza sin tener en cuenta la afectación que podrían 
desencadenar los fenómenos naturales. En este sentido, desde el riesgo también se construyen imaginarios urbanos, pues se interviene el espacio para posibilitar algunas condiciones para el habitar.

Es decir, se destacan los nexos entre las fluctuaciones de orden económico, físico y social que complejizan la gestión del riesgo como instrumento que opera en las comunidades y que debe abordarse, sin perder de vista el carácter físico de la amenaza y vulnerabilidad antes escenarios de fenómenos naturales, pretendiendo un hábitat para albergar y construir sus proyectos de vida.

Por lo que la perdida de lazos con el territorio, a partir de un desplazamiento hacia otro lugar de residencia, se considera como el desprendimiento de la participación ciudadana, la tenencia de los bienes que se han conseguido por medio del habitar y la fragmentación de la historia en el asentamiento que, les permitió aferrarse a una tenencia no segura de la tierra. De igual forma, el sentido de incertidumbre ante la llegada e inserción también puede generar tal desarraigo del nuevo lugar, en el que se desarrollará el habitar.

Por consiguiente, las operaciones urbanísticas como la del reasentamiento, pasan a ser un elemento fundamental, pues posibilitan la formación de vínculos al interior de la sociedad; adicionalmente tiene la capacidad de transformarlos. Es decir, puede potencializar las particularidades y características ya existentes dentro de las comunidades, de acuerdo con los lazos que ya se han creado; configurando nuevas dinámicas urbanas. De allí la importancia de crear un vínculo para la planeación (Comunidad-Administración pública).

Con ello, la provisión de viviendas de reasentamiento se ha convertido en un instrumento de gestión del territorio, incidiendo en las condiciones de vida de las poblaciones que, habitan zonas en riesgo, o que engrosa las cifras de déficit de vivienda, sin que por ello se reconozcan las afectaciones. Dicho así, los programas se implementan sin tener en cuenta las diferentes variables que se desarrollan en el territorio, por lo que los métodos de producción y existencia en el espacio se modifican, produciendo inequidad social al momento de decidir, quiénes deben quedarse en el riesgo y quiénes deben trasladarse.

Asumiendo las nociones sobre reasentamiento y de la necesidad de repensar este programa, es necesario precisar el reasentamiento colectivo como: la autoproducción del espacio habitable de una comunidad, en la que se tejen relaciones de vecindad, comunitarias e identitarios que, permiten conservar códigos colectivos y modos de habitar, para el devenir de la producción social del hábitat en un traslado. Por lo que 
este tipo de acciones desde la administración pública promovería niveles de calidad de vida y relacionamiento con el medio físico-natural, fundado en la autoproducción de la vivienda y el entorno, en todas las fases del traslado de la población para asentamientos que se ubican en zonas afectadas por riesgos socio-naturales.

Otro discusión, se da en torno al ordenamiento territorial, pues no existe una definición del reasentamiento, ya que este es mencionado tangencialmente en la normatividad del país, utilizando términos de reubicación y reasentamiento indistintamente, para hacer referencia de la obligación y el papel de las autoridades locales de trasladar personas que, se encuentren en zonas de riesgo, sin tener un marco de referencia más allá del hábitat como vivienda y el mejoramiento de vida, asociado a evitar la ilegalidad en la tenencia de vivienda, lo que resta herramientas a los entes territoriales frente a la capacidad de acción.

Entonces, la gestión local debe entender los imaginarios y procesos sociohistóricos que han ocurrido en el espacio habitado, por ejemplo, para algunos habitantes el riesgo no existe, porque el fenómeno no es amenazante y porque su vida ha girado en torno a esa porción del espacio, en donde han establecido formas de vida y redes de cuidado ante un evento amenazante.

A diferencia de la política de gestión de riesgo institucional que, atiende las emergencias y desastre con un carácter asistencialista, donde se trata a la población como cifras y no como víctimas que, pretenden una reparación integral en su calidad de vida, lo que permitiría entender que el reasentamiento, va más allá de una vivienda de llegada, pues se deben tener unos mínimos de habitabilidad, espacio público y derecho a la ciudad, teniendo en cuenta lo que mencionan Hurtado y Chardón (2012), la relación del ser humano con el medio habitado que, permite la permanencia a partir de sus múltiples dimensiones.

\section{Soporte de la materialidad del hábitat}

En segundo lugar y con el ánimo de reconocer experiencias que han sido limitadas, a partir del traslado poblacional, se expondrá el caso de estudio, teniendo en cuenta las categorías propuestas anteriormente, desde la autoproducción del hábitat en la ocupación en el barrio Caracolí, llevado a cabo en el año 2011, este tiene la característica de ser individual y dispersor de la población en distintos proyectos de vivienda. El barrio Caracolí se ubica en la UPZ 69 Ismael Perdomo-Bogotá, esta se caracteriza por tener accidentes geográficos de alta variabilidad, lo que implica que además de pendientes pronunciadas, discurren cuerpos hídricos que proponen una amenaza a partir de la trasformación antrópica del espacio habitable. 
La ocupación se gesta a partir de 1994, por parte de los denominados "tierreros", que, en alianza con grupos al margen de la ley, se valen de vacíos legales y carencia en las delimitaciones espaciales, para apropiarse de suelo de expansión urbana o de protección, lotearlos y comercializarlos de manera fraudulenta. Con el transcurrir del tiempo y la cotidianidad, se produjeron espacios comunes, mejoraron y adecuaron redes de servicios públicos, lo que se tradujo en la legalización del asentamiento en 2007, a pesar de ello, más habitantes siguieron ocupando las laderas, lo que promovía sobrecarga del suelo.

Figura 1. Asentamiento de origen informal Caracolí en Bogotá-Colombia. Autoproducción de vivienda.

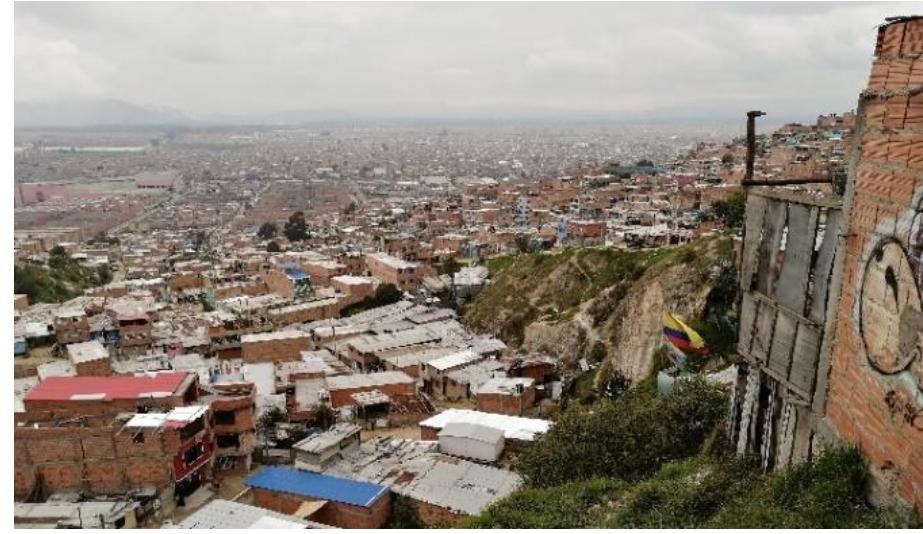

Fuente: Elaboración propia. Jeison Andrés Hincapié Rodríguez. 2020.

Según Torres (2011), el 29 de abril de año 2010, se presenta un deslizamiento, generando pérdidas de vidas y la destrucción de viviendas, este llevó a la Caja de Vivienda Popular-CVP (Institución que tiene a cargo realizar los procesos de reasentamiento) a iniciar el proceso de reasentamiento en el mes de mayo del mismo año, como se observa en la figura 2. Es importante mencionar que el barrio no se reasentó en su totalidad como se observa en la figura 3, así la población que se reasento fue un total de 154 familias, dispersadas en los barrios: El Sol-Ciudadela Nuevos Usme, El Bicentenario (Bosa) y Colores de Bolonia I (Usme), objeto de estudio del presente documento. 
Figura 2. "La casa se nos vino encima".

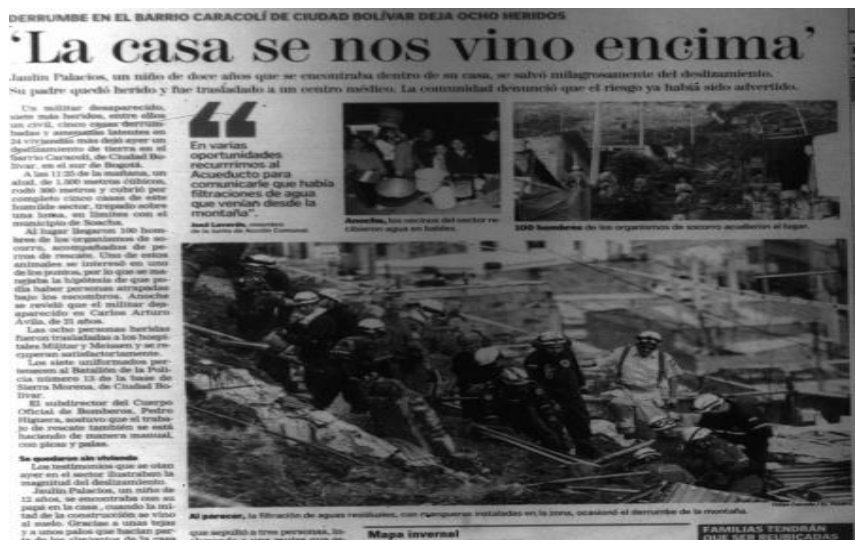

Fuente: (El Tiempo, 2010) Nota de prensa del periódico El Tiempo. En ella se enuncia el deslizamiento en el barrio Caracolí del 29 de abril de 2010, luego de ello sucederían nuevos deslizamientos que conducirían a declarar el riesgo inminente.

Figura 3. Barrio Caracolí-Bogotá.

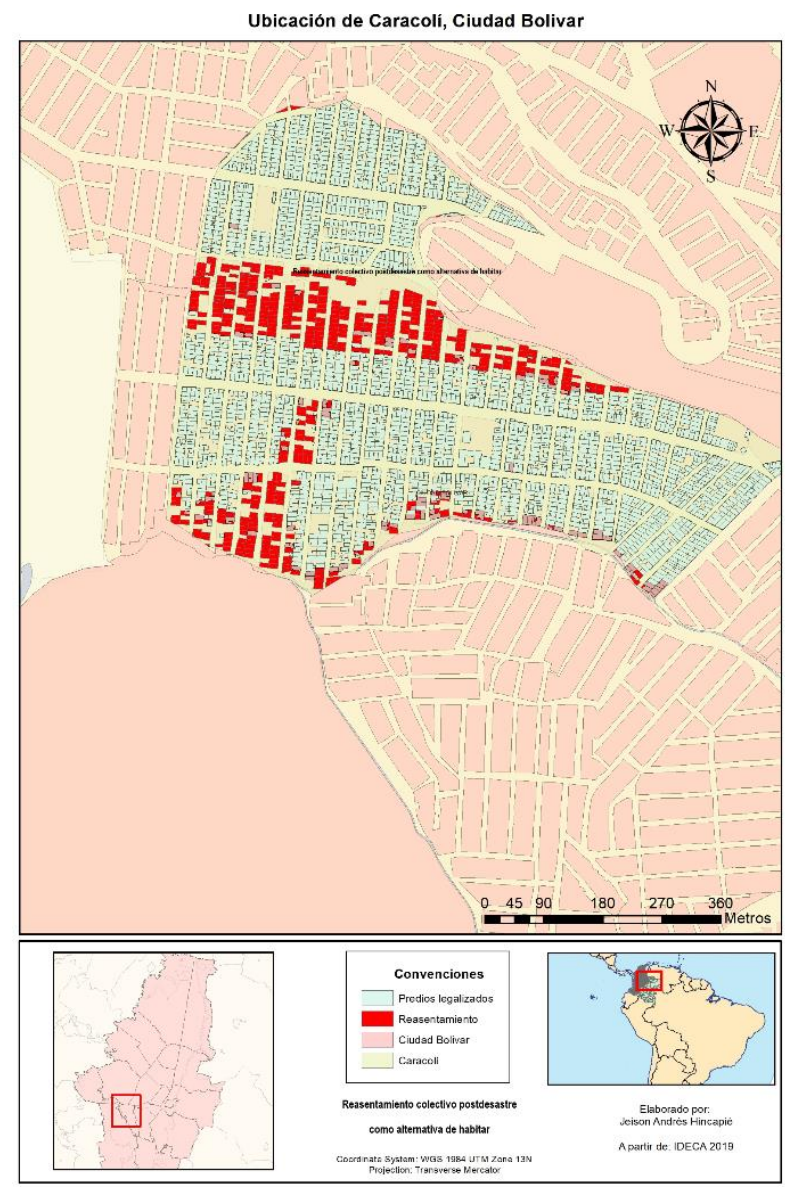

Fuente: Elaboración propia. Jeison Andrés Hincapié Rodríguez. 2020.

Luego de que la CVP ofreciera por medio del valor único de reconcomiendo VUR, las familias eran trasladadas a un hábitat transitorio, para que luego de un proceso de 
adjudicación y avalúo se realizara un traslado definitivo. Es así como, 154 familias fueron dispersadas en proyectos de Vivienda de interés social VIS, en Bogotá y en otros entes territoriales.

Para el presente caso, se referenciará Colores de Bolonia I, el cual es un proyecto de vivienda de interés social (Se caracterizan porque su valor no puede ser superior a 17.300 USD) que entregó 312 viviendas, de $42 \mathrm{~m} 2$ del área total de lote, las cuales constan de 2 habitaciones, baños, sala-comedor, cocina. Se ubica en la cuenca de la quebrada Santa Librada, lo que implica riesgos de inundación, además de estar asentado en pendiente (Ver figura 4 y 5), para la cual se hizo relleno, previendo su estabilización y posterior construcción, a este barrio se reasentaron 23 familias de Caracolí.

Figura 4. Relleno y pendiente en Colores de Bolonia I.

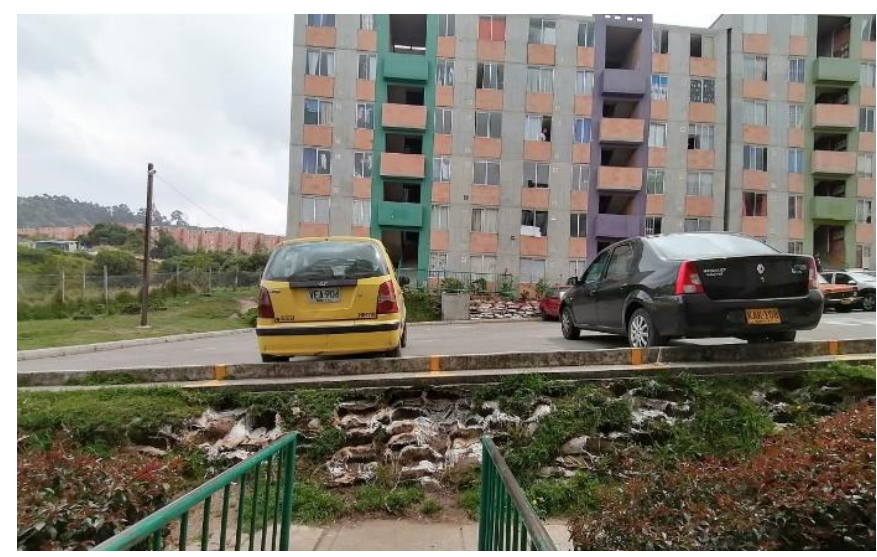

Debido a la pendiente donde se construyeron las nuevas viviendas, fue necesario rellenar el suelo.

Fuente: Elaboración propia. Jeison Andrés Hincapié Rodríguez. 2020.

También, es importante mencionar que este barrio fue entregado en el año 2015, por lo que sus destinarios finales, se vieron obligados a habitar transitoriamente, mientras les entregaran las unidades habitacionales. 
Figura 5. Ubicación Colores de Bolonia I-Usme. Bogotá.

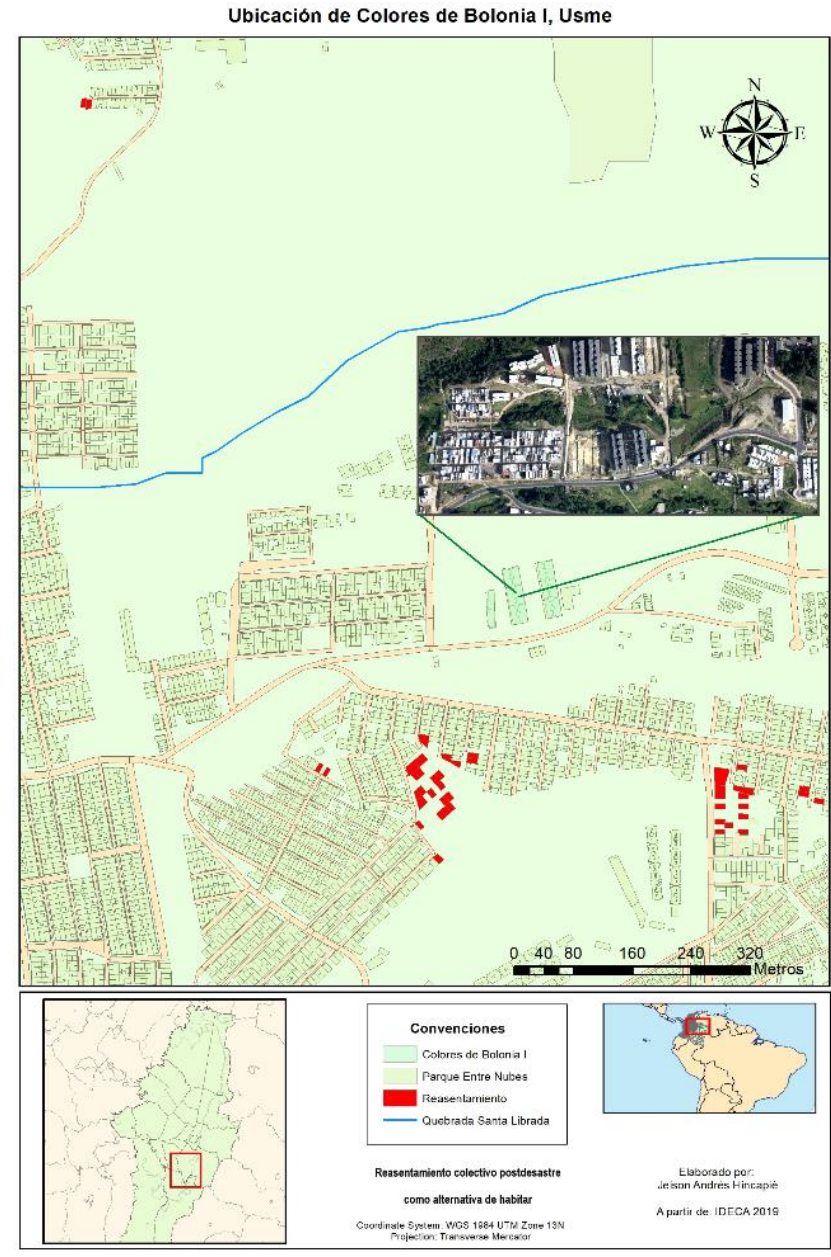

Fuente: Elaboración propia. Jeison Andrés Hincapié Rodríguez. 2020.

Además, se presentan grandes inconvenientes de habitabilidad, debido a la calidad en materiales, ubicación y tipología, lo anterior teniendo en cuenta la visita a campo, así como el grado de pendiente y la cercanía a los cuerpos hídricos que rodean el proyecto de vivienda. También, la totalidad de las familias, han tenido un aumento significativo en pago de servicios y contribuciones en impuestos y demás, pues el estrato de las viviendas nuevas es 2, a diferencia de Caracolí, que luego de 2007 era 1. (estratos que se conforman en orden ascendente a partir del valor del suelo, ubicación y acceso a servicios y equipamientos)

Esta ampliación de los gastos, sumado a otro factor de la propiedad horizontal, como lo es el pago de administración, han implicado problemáticas económicas y sociales. Así, las familias se encuentran ante una vulnerabilidad importante, pues se pueden pagar los servicios, pero no las adecuaciones u otros gastos. Promoviendo así procesos de segregación y empobrecimiento. 
Por su parte, frente a la disposición de equipamientos, servicios y espacios públicos, se evidencian dificultades, lo que implica impactos directos e indirectos en el sistema amplio del hábitat en construcción y consolidación de la participación, que se encargan de la búsqueda de mejores condiciones del entorno. Entonces, sí hay una transformación que valoran como positiva las familias consultadas, ello por la seguridad en la tenencia de la vivienda, diferente a la condición previo al reasentamiento; sin embargo, esta resolución del hábitat de manera integral está permeada por las relaciones que aumentan las tensiones, que a la vez promueven una carencia de cohesión territorial.

Sin embargo, se debe agregar que el hecho de recibir la vivienda como un valor de cambio y desde una perspectiva eminentemente física, impide la consolidación del proceso de habitar y mientras que las viviendas son entregadas sin acabados, bajo el discurso "para que las familias las adecuen como quieran", desconociendo que los recursos son limitados. Además, para el día 18 de diciembre de 2019, se presentó una inundación, lo que afectó a los primeros pisos de los apartamentos, mientras que los residentes aseguran que no es la primera vez que sucede, que han solicitado respuesta a la constructora y a la CVP sin que exista alguna intervención.

Lo que además de la reducción simplista, de ver la dimensión física de la vivienda, implica nuevas condiciones de vulnerabilidad, lo que resta legitimidad al proceso, mientras genera nuevas preocupaciones para quienes fueron reasentados. Por lo que se presentan grandes inconvenientes de habitabilidad, debido a la calidad en materiales, ubicación y tipología, lo anterior teniendo en cuenta la visita a campo, así como el grado de pendiente y la cercanía a los cuerpos hídricos que rodean el proyecto de vivienda.

Figura 6. Llevo mi casa conmigo.

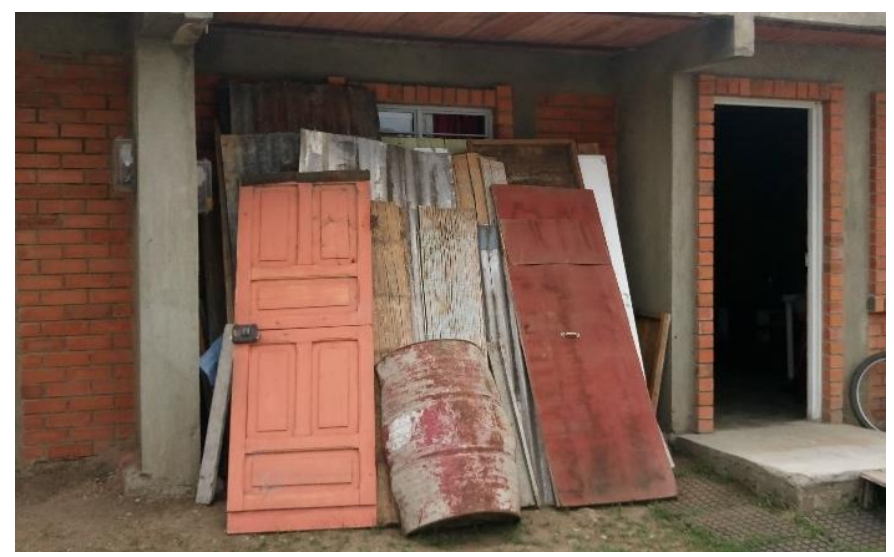

En la imagen se observa ornamentación recuperada por una familia, luego del desalojo y reasentamiento, la cual adecuan en sus nuevas viviendas. Fuente: Elaboración propia. Jeison Andrés Hincapié Rodríguez. 2020.

Vol. XV - Año 2021 - pp.8 - 29 - Artículo libre - Proyección 
Entonces, este ideal de la vivienda implica seguridad; sin embargo, dista de una vida imaginativa, cargada de emociones y experiencias estéticas, no solo en la consolidación de la vivienda, sino en la conformación del hábitat, a partir de la adecuación del habitar, como se observa en la figura 6 . Es importante lo que mencionan las personas respecto a lo físico de la vivienda; sin embargo, agregan que la historia que los antecede, dista de los nuevos hábitos que componen su esencia y existencia en los barrios de llegada, en el que la nueva economía ha impedido la producción del habitar común.

Así, el hábitat integral influye de manera directa en la sostenibilidad de los reasentamientos, en términos sociales, convivencia y construcción de colectividad, en aspectos físicos debido a las nuevas condiciones de las viviendas y su entorno, desde la producción de espacios para vivir; los cuales sumados a las maneras en cómo se concibe ahora su mundo, construyen nuevos modos de habitar, teniendo el precedente de no habitar, por los factores expuestos.

\section{Autoproducción del hábitat para reasentamientos colectivos}

De acuerdo a lo anteriormente expuesto, en el tercer aparto se plantean lineamientos para el reasentamiento colectivo, ello a partir del trabajo de observación participante y de los grupos focales realizados con los habitantes de Colores de Bolonia I. Los cuales denotaron reducción de su calidad de vida, a partir de la disminución en metros cuadrados ocupados (Por lo que se han hecho adecuaciones fraudulentas, aunque son fundamentales desde la forma en como una vivienda desde la esfera privada, se abre al mundo a partir de una extensión hacia lo público), carencia de iluminación y ventilación; así como oferta en espacio público, equipamientos, líneas de transporte cercanos y nuevas amenazas.

En consecuencia, la vida de quienes participaron de la investigación ha cambiado, hay factores como los mencionados que se juntan con la ubicación en periferia, perdida de redes sociales, aumento del costo de vida y una mayor inseguridad, implicando así una difícil adaptación en la que, se construye un hábitat bajo los preceptos de la necesidad. Es fundamental asumir que la organización, en una fase posterior a los barrios de llegada ha sido mínima, lo que implica otra serie de vulnerabilidad social urbana, caracterizada, entre otras por, la ubicación de las comunidades en la trama urbana, la calidad del hábitat, el nivel de organización de la comunidad, los proyectos y programas realizados; así como el acceso a equipamientos que ofrece la ciudad.

Así pues, el trabajo colaborativo con la comunidad, promovió que los lineamientos se organicen así: en primer lugar, el eje que se denomina preparando el reasentamiento colectivo, en el que se plantea trabajar en los hábitos cotidianos y repetitivos, el territorio, 
sus identidades individuales y colectivas, comprendiendo y potenciando la participación de las personas a reasentar, facilitando las nociones de hábitat desde las viviendas y luego en el exterior, ya que, en el trabajo de campo, las familias manifestaron resistencia frente al proceso de compra de las viviendas, pues no se hicieron ofertas que tuvieran en cuenta las adecuaciones físicas y la representación de cada una de ellas en su contexto próximo.

Entonces el avaluó de las viviendas y dejar el territorio es una fase de choque entre la comunidad y el Estado, por lo que las entidades encargadas de esta fase, deben cambiar el paradigma con el que se ha contemplado el hábitat urbano, en el que se relacionen las dinámicas externas a las unidades físicas, dando valor y sentido a los códigos generados durante la autoproducción de la vivienda y su adecuación al medio.

Es importante mantener el tejido social de procedencia, pues conservaría un nivel de cohesión ante un hecho que vulneró su habitabilidad en un momento dado. Procurando con ello, que se conozcan y que la relación con el territorio de llegada sea más fácil, pues habría un sentido de comunidad, que deberá ser analizado desde las estructuras, los actos cotidianos y las coyunturas que se atraviesan, en Colores de Bolonia I. Por su parte, el sentido e importancia de la cotidianidad, radica en como lo señala Lindón, (2004) que a partir de esta se tejen los modos de habitar los lugares y el reconocimiento de colectividad y sujeto social.

Luego, en el segundo eje usos y funciones en el espacio para consolidar un hábitat, se pretende producir un hábitat que responda a las necesidades de familias en condición de reasentamiento, lo cual debe ser sostenible, para evitar un posible desplazamiento y retorno a los barrios de salida. Por lo tanto, se deben integrar diversas entidades desde las prestadoras de servicios, de integración social, planeación, hasta constructores y comunidad, develando sus particularidades multidimensionales.

El conjunto de acciones debe responder a resolver las necesidades inmediatas, aspiraciones y expectativa de los pobladores, brindando a las organizaciones y comunidad no organizadas, una postura decisiva, que interlocute con las distintas entidades del ente territorial, para la producción social de su nuevo hábitat, buscando el interés colectivo, como se puede evidenciar en la figura 7, a partir de un ejercicio que se realizó con la comunidad. 
Figura 7. intervención en los espacios comunes de Colores de Bolonia I.

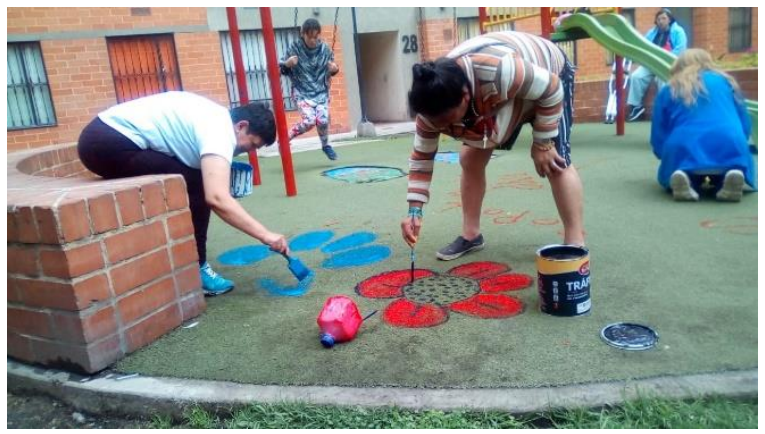

Fuente: Elaboración propia. Jeison Andrés Hincapié Rodríguez. 2020.

Este se puede comprender desde la calidad de las viviendas reasentadas, las que deberán comprender particularidades de la población, tales como condiciones laborales, de desplazamiento, físicas, entre otras, para que la respuesta sea efectiva, reduciendo la presencia de humedad, crear rampas de acceso, tener en cuenta patologías de salud preexistentes y el número de personas para evitar condiciones de hacinamiento, entre otros.

En términos del exterior de la vivienda, los equipamientos y servicios deben responder a las lógicas colectivas de la población reasentada, pues la configuración del espacio social está compuesta por los hechos sociales, relacionados con el nuevo hábitat, lo que también deberá reconocer viviendas productivas, contemplando la posibilidad de tener pequeños negocios y promoción de la diversidad para la apropiación del hábitat.

El tercer eje se denomina producción social del hábitat, pues de acuerdo con Ortiz (2007), existen elementos claves los cuales circundan en los ámbitos de lo colectivo y privado, relacionados a partir de un proyecto político, a los hábitos individuales y colectivos, que se transforman a partir del espacio-tiempo y sus dinámicas, las que van generando identidad, creación del territorio. Estas se sostienen a su vez, en la economía solidaria como formas de intercambio cercano y posible; la participación de los ciudadanos como respuesta a las tensiones sociales que pueden darse en proceso que homogenizan los discursos del hábitat y lo reducen a la vivienda, todo ello como manera de facilitar la producción del hábitat.

La producción social del hábitat es indispensable luego de un proceso de reasentamiento, es determinante para el éxito del programa y de la construcción de ciudad a mediano plazo. Por lo que se requiere de una participación comunitaria importante, para que se concerté junto a la administración pública una gestión, ordenamiento y financiación del hábitat que se ocupa, por lo cual se señalan algunas prácticas que son reconocidas tanto por los funcionarios entrevistados, como por la 
población de los cuatro barrios que participaron, es fundamental mencionar que el proceso productivo debe controlarse desde la población, y compartirse con la asesoría por parte de los entes, ver figura 6.

Figura 8. Con el fin de construir los lineamientos, se realizaron taller de co-gestión con la comunidad del barrio Colores de Bolonia I.

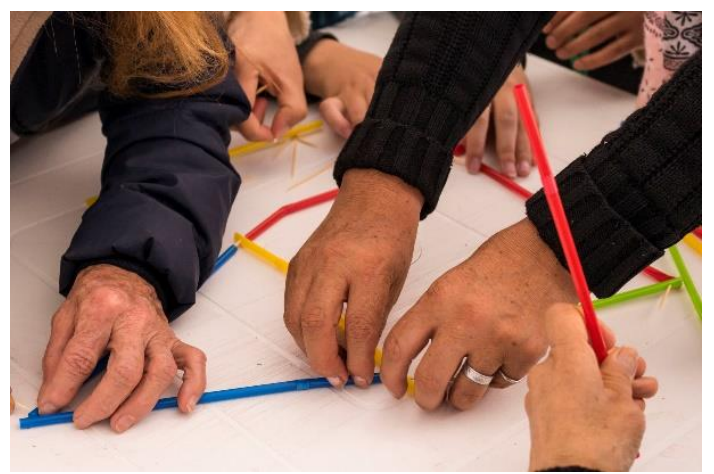

Taller de producción de hábitat, grupo focal en Colores de Bolonia I. Fuente: Elaboración propia. Jeison Andrés Hincapié Rodríguez. 2020

En tal manera, la producción del hábitat se sustenta sobre procesos de planeación y acción, a partir de las necesidades de la colectividad, es decir una participación decisoria para modificar el habitar de manera integral. Con ello, poder establecer maneras de intervenir, desde la construcción colectiva que reconozca diferencias, mientras gestiona desde la multiplicidad de las experiencias previas, para enfrentar los escenarios que tienen lugar en el nuevo espacio habitable, lo que puede aumentar o disminuir las tensiones.

Estas estrategias además de reducir costos, debido a la co-creación y el trabajo comunitario, promueven la utilización de espacios y materiales propios de los destinatarios. Quienes desarrollan innovación social como estrategias para mejorar las condiciones de su nuevo hábitat, mientras que se genera un sostenimiento para la apropiación, adaptación al contexto y nuevos modos de habitar, bajo el control de los mismos habitantes en reasentados.

Por último, el eje de consolidar el papel de los diferentes actores, con el ánimo de promover que la gobernanza del hábitat como un eje transversal, donde las capacidades de las instituciones y del personal, se ha transformado a partir de los nuevos requerimientos en materia de reasentamiento en sus distintos escenarios, como son la gestión, deliberación, participación de la comunidad y gestión integral para el reasentamiento. Ello desde una lógica facilitadora, no impositiva, ni intervencionista donde se desconozcan las amplias y diversas formas de los territorios, bajo lógicas de autoproducción para la organización comunitaria y la resolución de tensiones. 
También, será fundamental la asesoría, para la postulación a proyectos con recursos, que aumenten las acciones en torno a la mejorar del hábitat, así como pedagogía para el uso adecuado de la economía comunitaria, a fin de optimizar la gestión. Facilitar, por medio de instrumentos de gestión del suelo, programas que incluyan equipamientos para el mejoramiento de la vivienda, el entorno y las escalas zonales, lo que incluye mejorar la entrega de servicios, transparencia y auditorias constantes. La participación por parte de la comunidad procurará diseñar proyectos que hagan sostenible el hábitat, representación de las necesidades próximas, tendiendo a su integración horizontal con la administración local.

De igual manera, realizar un avaluó social donde el carácter de lo intangible también tenga valor para el pago del subsidio o compensación, el cual tendrá como base una la producción previa del hábitat. Además, de posibilitar una mayor cohesión social y promover una mejorar calidad de vida a partir de un escenario de reasentamiento, pues a partir de dicha intervención lo que se pretende es la mejorar en las condiciones de vida y no postergar la vulnerabilidad social.

Además, como mecanismo de gestión del suelo, y teniendo en cuenta la ley 388 de 1997, es necesario un fortalecimiento del banco de tierras de los municipios, con miras a presentarle a las habitantes opciones de reasentamiento colectivo, lo que además de posibilitar una mayor apropiación social, permitirá controlar los precios del suelo y con ello, el final de la vivienda, consolidando así un mercado inmobiliario controlado por el municipio.

\section{Conclusiones}

En el sentido del objetivo del documento, abordado desde un estudio práctico y luego la propuesta de lineamientos, elaborados a partir del trabajo comunitario desde grupos focales y observación participante, se concluye que el reasentamiento, no puede ser elaborado a partir de datos netamente cuantitativos y de la visión reduccionista del diseño urbano tradicional; por el contrario, debe ser el resultado de representaciones, construcciones y concepciones, en este caso, de las formas en cómo se vive y disfruta el espacio público; además, depende de un contexto y tramas sociales establecidas y constantemente re-legitimadas en los barrios de llegada.

Reconociendo que los reasentamientos han tenido un enfoque asistencialista que, resta la participación de los ciudadanos en la producción de su propio hábitat, el cual entra en conflicto con la complejidad de la provisión de la vivienda que, no tiene en cuenta las dimensiones sociales, económicas, ambientales y políticas, lo que pone en riesgo la sustentabilidad de los barrios reasentados. Es decir, mientras no existan unos 
instrumentos precisos que fortalezcan la participación de la ciudadanía en su construcción, no se tendrán reasentamientos que respondan a las necesidades de los territorios y sus pobladores.

La organización barrial ha sido difícil de implementar y mantener, debido a las nuevas lógicas con la que se deben enfrentar en un barrio formal, a lo que se suman las normas de propiedad horizontal, que además va de la mano con el hecho de que los lazos colectivos fueron fraccionados, dificultando aún más el poder acoplarse a un nuevo espacio habitable. Entonces, los efectos contrarios en la planeación del reasentamiento, está impidiendo que el programa institucional, tenga éxito y sostenibilidad en el tiempo, con lo que se prolonga una condición de exclusión a la ciudad, a partir de distintos ámbitos y esferas.

Reflejado en la continuidad de la población en áreas periféricas de la ciudad, manteniendo la segregación y marginación, que además de dificultar el acceso a bienes y servicios urbanos, sugiere dificultades en la movilidad, sobre todo de algunos grupos sociales, para los que no se realiza un diseño urbano y mobiliario especifico, ya que se homogenizan las soluciones habitacionales y sus entornos.

Teniendo en cuenta el sentido del hábitat, este tiene características de ser integral, por lo que aborda la vivienda desde la interioridad y desde el entorno, mientras que se sostiene en componentes físicos y sociales, por lo que es necesario ir más allá de la ocupación física de un territorio, es decir del proceso en la producción. Como se mencionó en el segundo apartado, para la sostenibilidad de los reasentamientos, en términos sociales, convivencia y construcción de colectividad, en aspectos físicos debido a las nuevas condiciones de las viviendas y su entorno, desde la producción de espacios para vivir; los cuales sumados a las maneras en cómo se concibe ahora su mundo, construyen nuevos modos de habitar, teniendo el precedente de no habitar, por los factores expuestos.

En tal forma, el hábitat está dotado de características simbólicas de la existencia, para este caso, en asentamiento informales que implican una serie de externalidades sociales, políticas, economías, ambientales, entre otras; desde distintos hábitos que se acoplan al espacio-tiempo, en la medida que se producen identidades colectivas y de distintas escalas, implicando así la multiplicidad de los actores en el espacio vivido, que las administraciones pública no han otorgado significancia, más allá de preservar la vida de quienes se ven expuestos al riesgo.

Por lo tanto, la participación de la comunidad es pilar para la producción del hábitat, desde sus perspectivas, lógicas y necesidades que, los programas se han limitado al 
componente físico instrumental, obviando los aspectos histórico-sociales e imaginarios de la existencia misma; así como dimensiones políticas, ambiental, y culturales. Por lo que los procesos colectivos de autoproducción de las viviendas, infraestructuras y servicios comunales, implica asumir un papel protagónico frente a los derechos a la comunidad, vivienda y a la ciudad. Adicionalmente la cercanía y frecuencia de los lazos comunitarios, superarán las condiciones adversas de un proceso de reasentamiento por desastre, el que tiene el carácter de involuntario.

Es prudente entonces promover una gobernanza del hábitat, a partir del reconocimiento de la diversidad social y cultural de los asentamientos de origen y sobre los polígonos de recepción del reasentamiento. Ello plantea reconocer los modos de habitar de las comunidades, de manera individual, familiar y del barrio, para no generar rupturas en las tramas de vida y producción social en el espacio habitable. Por lo que el reasentamiento colectivo, promueve los niveles de calidad de vida y relacionamiento con el medio físico-natural, a partir de la participación y decisión de la comunidad en todas las fases, desde el la declaratoria de riesgo, hasta el traslado a asentamientos que se ubican en zonas afectadas por riesgos socio-naturales.

Siendo indispensable un acompañamiento por parte de la academia, movimientos sociales y otros actores, que potencien los encuentros y conformación de nuevas prácticas, sin que esto implique el hecho necesario de que las comunidades en sí mismas, no puedan construir sus nociones de hábitat; finamente, las instituciones serán facilitadoras para promover las distintas dimensiones que del hábitat hagan parte. 


\section{Referencias bibliográficas}

ARRIETA, L. (2005). Identificación de estudios y acciones necesarias para la formulación del nivel individual de una política de reasentamiento en el Distrito Capital. Informe final, Secretaría de Hacienda Distrital - PNUD, Bogotá.

CERNEA, M. (1995) Understanding and preventing impoverishment: the state of knowledge in resettlement. Journal of refugee studies. Volumen 8. Pp 245-264.

CHARDÓN, A. (2010). Reasentar un hábitat vulnerable. Teoría Vs práxis. Revista INVI. Instituto de la vivienda. Facultad de arquitectura y urbanismo. Universidad de Chile. Volumen 25. Número 70.

CONTRERAS Y. Y BELTRÁN, M. (2015) Reconstruir con capacidad de resiliencia. El casco histórico de la ciudad de constitución y el sitio de desastre del terremoto y Tsunami del 27 de febrero de 2010. Revista INVI. Número 83, mayo 2015. Volumen Número 30. Universidad de Chile. Santiago de Chile.

CORREA, E. (1997). Impactos socio-económicos de grandes proyectos: evaluación y manejo. Bogotá: Fondo FEN.

ECHEVERRÍA, M. (2009). Hábitat: Concepto, campo y trama de vida. En M. C. Echeverría, ¿Qué es el hábitat?: la preguntas por el hábitat. Medellín: Facultad de arquitectura. Universidad Nacional de Colombia. Sede Medellín.

ECHEVERRÍA, M Y RINCÓN A. (2000). Ciudad de territorialidades Polémicas de Medellín. Medellín: Centro de Estudios del Hábitat Popular - CEHAP. Universidad Nacional de Colombia-Sede Medellín.

HURTADO J. (2010) Reasentamiento y habitabilidad. De los asentamientos autogestionados a las soluciones institucionales masivas de vivienda. Estudio de caso, barrio Altos de Santa Ana, comuna Ciudadela del Norte, Manizales, Tesis de maestría en hábitat. Universidad Nacional de Colombia-Manizales.

HURTADO, J. Y CHARDÓN C. (2012) Vivienda social y reasentamiento, una visión crítica desde el hábitat. Universidad Nacional de Colombia sede Manizales.

LINDÓN, A. (2004) Las huellas de Lefebvre sobre la vida cotidiana. Revista veredas pp 39-60. 
ORTÍZ, E. (2007) Producción social de vivienda y hábitat: bases conceptuales para una política pública. En. El camino posible: producción social del hábitat en América Latina. Montevideo Trilce.

PATRIDGE, W. Edit. (2000), Reasentamiento en Colombia, Banco Mundial. Red de solidaridad social ACNUR. Corporación Antioquia Presente, Tercer Mundo editores.

SERJE, M. (2011) Los dilemas del reasentamiento. Introducción a los debates sobre procesos y proyectos de reasentamiento. En, Serje, M. y Anzellini, S. (2011) Los dilemas del reasentamiento, debates y experiencias de la mesa nacional de reasentamiento de población. Bogotá, Universidad de los Andes.

TORRES, C. (2011) Producción y transformación del espacio residencial de la población de bajos ingresos en Bogotá en el marco de las políticas neoliberales (1990-2010) Una aportación a la comprensión del problema de la vivienda a través de cuatro casos de estudio de la localidad de Ciudad Bolívar. Tesis doctoral. Valladolid. Instituto Universitario de Urbanística.

Abreviaturas

CVP: Caja de vivienda popular

VIS: Vivienda de interés social 